\title{
EZH2 suppresses hepatocellular differentiation of mouse bone marrow mesenchymal stem cells
}

\author{
T. Lu' ${ }^{1}$, H. Sun ${ }^{1}$, J. Lv ${ }^{1}$, M.F. Yang ${ }^{1}$, F. Zhang ${ }^{1,2}$, Y. Qian ${ }^{1,2}$ and X.J. Dong ${ }^{1}$ \\ ${ }^{1}$ Clinical Laboratory Center of Shaoxing People's Hospital, \\ Shaoxing Hospital of Zhejiang University, Shaoxing, China \\ ${ }^{2}$ School of Laboratory Medicine and Life Science, \\ Wenzhou Medical College, Wenzhou, Zhejiang Province, China \\ Corresponding author: Xuejun Dong \\ E-mail: dxj9666@163.com
}

Genet. Mol. Res. 13 (1): 2231-2239 (2014)

Received January 8, 2013

Accepted November 18, 2013

Published March 31, 2014

DOI http://dx.doi.org/10.4238/2014.March.31.3

\begin{abstract}
Our previous studies have indicated that mouse bone marrow mesenchymal stem cells (mBMMSCs) have potential to differentiate into hepatocytes with high efficiency. Our study aimed to evaluate the role of the mouse histone methyltransferase enhancer of zeste homolog 2 gene (EZH2) in the hepatocellular differentiation of mBMMSCs. The mBMMSCs isolated from femurs and tibias were cultured in Iscove's modified Eagle's medium (IMEM) supplemented with $10 \%$ fetal bovine serum. Hepatocellular differentiation was induced by $20 \mathrm{ng} / \mathrm{mL}$ hepatocyte growth factor and $10 \mathrm{ng} / \mathrm{mL}$ fibroblast growth factor 4 . The mouse histone methyltransferase $E Z H 2$ gene was introduced via PLenti-eGFP-EZH2 or PLenti-eGFP-NEO as a control. Hepatocellular-induced mBMMSCs showed lower expression of EZH2 and lower level of histone $\mathrm{H} 3$ lysine 27 trimethylation (H3K27me3) in the $A F P$ and $F O X a 2$ gene promoter regions compared to uninduced mBMMSCs. Introduction of EZH2 inhibited hepatocellular induction, reduced both the mRNA and protein levels of AFP and FOXa2, and increased the level of histone $\mathrm{H} 3 \mathrm{~K} 27 \mathrm{me} 3$ in the AFP and FOXa2 gene promoter regions. In summary, the mouse histone methyltransferase
\end{abstract}


EZH2 gene could suppress hepatocellular differentiation of mBMMSCs by increasing the level of $\mathrm{H} 3 \mathrm{~K} 27 \mathrm{me} 3$ in the $A F P$ and $F O X a 2$ gene promoter regions.

Key words: mBMMSCs; Hepatocellular differentiation; EZH2; Histone methyltransferase

\section{INTRODUCTION}

Polycomb group proteins are key regulators of stem cell and cancer biology. They mainly act as repressors of differentiation and tumor-formation genes. Enhancer of zeste homolog 2 (EZH2), a core component of Polycomb repressive complex 2 (PRC2), functions as a methyltransferase for $\mathrm{H} 3$ lysine 27 trimethylation (H3K27me3) (Cao et al., 2002; Christophersen and Helin, 2010). Previous studies showed that H3K27me3 contributed to the maintenance of embryonic stem cell pluripotency (Boyer et al., 2006; Zhang et al., 2009). It was also reported that EZH2 played an essential role in the maintenance of both the proliferative and self-renewal capacity of stem cells. Over-expression of EZH2 completely conserved the long-term repopulating potential and restored the stem cell quality to normal levels in the transplantation of hematopoietic stem cells (Kamminga et al., 2006).

Recent studies have demonstrated that bone marrow mesenchymal stem cells (BMMSCs) can be induced to generate not only the progenies of mesodermal lineages, such as adipocytes, chondrocytes, and myogenic cells, but also cells from different germ layers, such as neuronal cells and hepatocytes. Our previous studies have indicated that mouse BMMSCs (mBMMSCs) have potential to differentiate into hepatocytes with high efficiency (Pan et al., 2008). We previously found that the histone deacetylase inhibitor valproic acid (VPA) could promote the hepatocellular differentiation of mBMMSCs. However, the role of EZH2 in the hepatocellular differentiation of mBMMSCs remains unclear.

In this study, we successfully constructed the mouse EZH2 gene lentiviral vector. We investigated the role of EZH2 in the hepatocellular differentiation of mBMMSCs, and found that over-expression of EZH2 could suppress the hepatocellular differentiation of mBMMSCs, possibly via increasing the level of $\mathrm{H} 3 \mathrm{~K} 27 \mathrm{me} 3$ in the $A F P$ and $F O X a 2$ gene promoter regions.

\section{MATERIAL AND METHODS}

\section{Mice}

Four- to eight-week-old male ICR mice, obtained from the Laboratory Animal Unit of Zhejiang Academy of Medical Sciences (Hangzhou, People's Republic of China), were used in the experiments. The animals were housed under specific pathogen-free conditions. All experimental procedures were performed according to institutional guidelines.

\section{Construction of the $E Z H 2$ lentiviral vector}

Polymerase chain reaction (PCR) was performed to amplify the mouse $E Z H 2$ gene based on the PCMV-SPORT6 vector recombinant EZH2 (Open Biosystem) with the KpnI and 
$X m a \mathrm{I}$ restriction site primers. The amplification product was cloned into the lentiviral vector PLenti-eGFP-NEO after being cleaved by KpnI and XmaI. The EZH2 vector was validated by restriction digestion and sequencing. The recombined vector was co-transfected into 293T cells with a four-plasmid system, pRsv-REV, pMDlg-pRRE, and pMD2G, to package the lentivirus particles.

\section{Isolation and culture of mBMMSCs}

mBMMSCs were harvested as described previously (Chen et al., 2007). Briefly, cells were obtained from the bone marrow of femurs and tibias of 8-week-old ICR mice, and cultured in Iscove's modified Eagle's medium (IMEM; Invitrogen Gibco, USA) supplemented with $10 \%$ fetal bovine serum (FBS; Tianhang Biological Technology, Hangzhou, China) and $1 \%$ penicillin/streptomycin (Invitrogen Gibco) at $37^{\circ} \mathrm{C}$ and $5 \% \mathrm{CO}_{2}$. Non-adherent cells were removed from plates $72 \mathrm{~h}$ later by changing the medium. At near confluence, the cells were re-plated at a density of 100 cells $/ \mathrm{cm}^{2}$.

\section{In vitro hepatic differentiation and $\mathrm{EZH} 2$ lentivirus infection}

BMMSCs of passage 3 were inoculated at $5 \times 10^{4}$ cells $/ \mathrm{cm}^{2}$ on 6 -well culture plates, and were divided into 4 groups. The mBMMSCs were cultured in IMEM. Hepatocellularinduced cells were treated with cytokines as described previously (Pan et al., 2008): hepatocellular differentiation was induced in IMEM supplemented with 10\% FBS, $20 \mathrm{ng} / \mathrm{mL}$ hepatocyte growth factor (Peprotech, Princeton Business Park, Rocky Hill, NJ, USA), and 10 ng/mL fibroblast growth factor 4 (Peprotech). The lentiviral vectors carrying the control gene and the $E Z H 2$ gene were added into the induction medium, respectively.

\section{Reverse transcription-polymerase chain reaction (RT-PCR)}

Total RNA was isolated using Trizol reagent (Invitrogen Gibco). The cDNA was synthesized using the PrimeScript ${ }^{\mathrm{TM}} \mathrm{RT}$ reagent kit (TaKaRa; Dalian, China) according to the manufacturer protocol. Quantitative PCR was performed using an SYBR PrimeScript ${ }^{\mathrm{TM}}$ RTPCR Kit (TaKaRa) in a real-time thermal cycler system. PCR reactions was carried out at $95^{\circ} \mathrm{C}$ for $30 \mathrm{~s}$ and then at $95^{\circ} \mathrm{C}$ for $5 \mathrm{~s}$, annealing at $60^{\circ} \mathrm{C}$ for $30 \mathrm{~s}$, and $72^{\circ} \mathrm{C}$ for $30 \mathrm{~s}$, for 40 cycles. The gene-specific primer sequences are provided in Table 1 . The $2^{-\Delta \Delta C T}$ method was used to analyze the data, where $\Delta \Delta \mathrm{CT}=\left(\mathrm{CT}_{\text {Target }}-\mathrm{CT}_{\text {Actin }}\right)_{\text {each group }}-\left(\mathrm{CT}_{\text {Target }}-\mathrm{CT}_{\text {Actin }}\right)_{\text {hepatic group }}$.

\begin{tabular}{|c|c|c|}
\hline Gene & Primer sequences $\left(5^{\prime}-3^{\prime}\right)$ & Product size (bp) \\
\hline$E Z H 2-F$ & TTTTATGGTGGAAGACGA & 425 \\
\hline$E Z H 2-\mathrm{R}$ & TACATTCAGGAGGCAGAG & \\
\hline$A F P-\mathrm{F}$ & CACTGCTGCAACTCTTCGTA & 300 \\
\hline$A F P-\mathrm{R}$ & CTTTGGACCCTCTTCTGTGA & \\
\hline FOXa2-F & GACCTCTTCCCTTTCTACCG & 551 \\
\hline FOXa2-R & TTGAAGGCGTAATGGTGC & \\
\hline$\beta$-actin-F & TTCCTTCTTGGGTATGGAAT & 200 \\
\hline$\beta$-actin- $\mathrm{R}$ & GAGCAATGATCTTGATCTTC & \\
\hline
\end{tabular}




\section{Western blot experiment}

Total protein extraction was carried out by harvesting $2 \times 10^{5}$ serum-deprived cells/ $\mathrm{mL}$ in ice-cold lysis buffer (Beyotime; Haimen, Jiangsu, China). The lysates were lysed in sodium dodecyl sulfate-polyacrylamide gel electrophoresis (SDS-PAGE) buffer, transferred to a polyvinyldifluoride (PVDF) membrane (BioRad; Hercules, CA, USA), and then was incubated with blocking solution for $1.5 \mathrm{~h}$. The membranes were incubated overnight at $4^{\circ} \mathrm{C}$ using specific antibodies against EZH2 (Abcam; USA), AFP (Santa Cruz Biotechnology; USA), FOXa2 (Santa Cruz Biotechnology), and $\beta$-actin (Santa Cruz Biotechnology). After three washes with phosphate-buffered saline with Tween (PBST), membranes were incubated with horseradish peroxidase-conjugated secondary immunoglobulin G (IgG; Santa Cruz Biotechnology) for $1.5 \mathrm{~h}$. Immunoreactive bands were detected using the ECL system (Beyotime).

\section{Chromatin immunoprecipitation assay}

Briefly, cross-linked chromatin was digested by micrococcal nuclease. The chromatin was immunoprecipitated using anti-H3K27me3 (Abcam) antibodies. Anti-RNA polymerase II antibody and normal rabbit IgG were used as the positive and negative control, respectively. Quantitative PCR was performed using the SYBR PrimeScript ${ }^{\text {TM }}$ RT-PCR Kit. The gene-specific primer sequences were: AFP promoter region (forward, AGCTGGCTCATCAGGTTT and reverse, CAGTAGTTCAGGCTATTCA); FOXa2 promoter region (forward, TGCTGCGT CTTCAGTCCA and reverse, GCCCAAATCCAGGTGTCC).

\section{Statistical analysis}

Statistical analysis was performed using SPSS version 16.0. All experiments were repeated at least three times, and data of evaluation parameters are reported as means $\pm \mathrm{SD}$.

\section{RESULTS}

\section{Recombinant PLenti-eGFP-EZH2 was cleaved by KpnI and XmaI}

Recombinant PLenti-eGFP-EZH2 was cleaved by KpnI and XmaI into two fragments: 7500 and $2241 \mathrm{bp}$ (Figure 1). The EZH2 gene was confirmed by sequencing.

\section{Morphological characterization}

mBMMSCs displayed typical fibroblast-like morphologies and visible uniform cell colonies (Figure 2A). Hepatocellular-induced cells showed typical liver cell-like morphology: polygonal and round cell colonies, clear nuclei, and granular cytoplasm (Figure 2B). Introduction of the control vector did not result in morphological changes to induce the differentiation of mBMMSCs (Figure 2C). On the other hand, introduction of PLenti-eGFP-EZH2 countered the effect of hepatocellular induction with most cells showing a fibroblast-like or fusiform morphology (Figure 2D). 

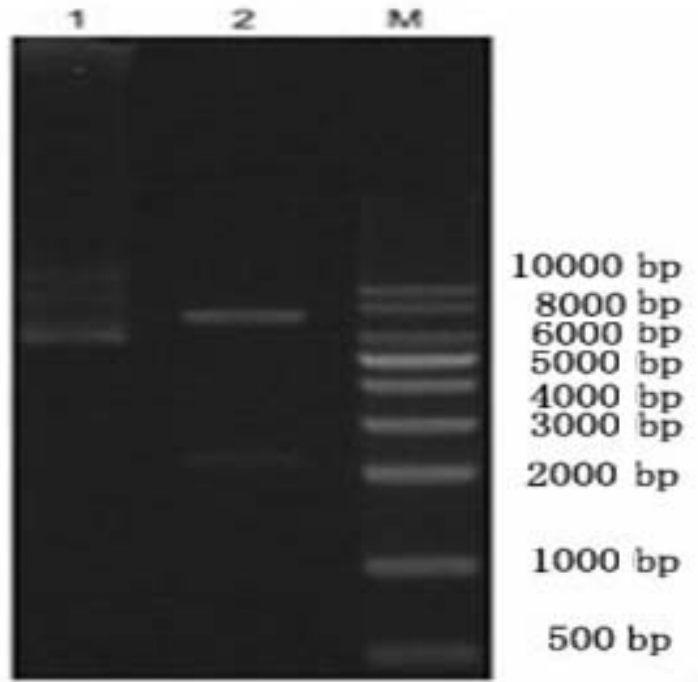

Figure 1. Restriction map of PLenti-eGFP-EZH2. Lane $M=$ marker DL 2000; lane 1 = PLenti-GFP-EZH2; lane 2 $=$ PLenti-GFP-EZH2 digested by KpnI and XmaI.

A

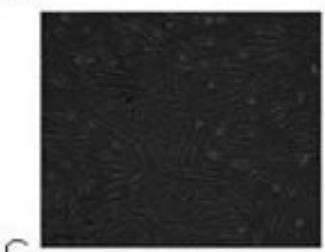

C

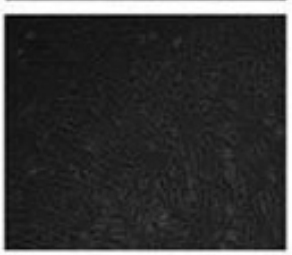

E

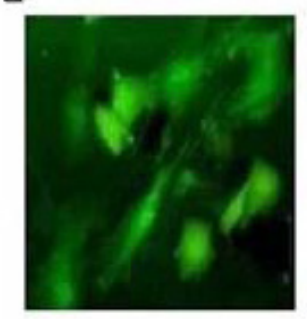

B

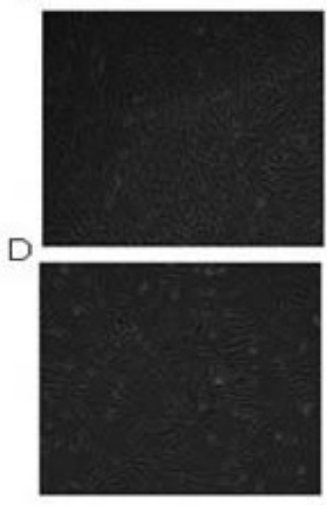

F

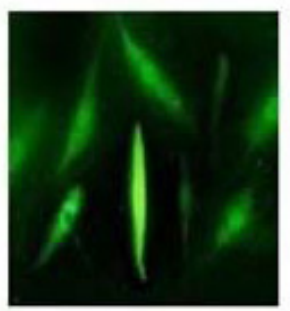

Figure 2. Morphological changes of different groups. A. mBMMSCs of passage 3 with no induction; B. mBMMSCs with hepatocellular induction; C. hepatocellular-induced mBMMSCs infected by PLenti-eGFP-NEO; D. hepatocellular-induced mBMMSCs infected by PLenti-eGFP-EZH2; E. and F. expression of GFP in the cells introduced with control vector and PLenti-eGFP-EZH2, respectively, under fluorescence microscope. 


\section{RT-PCR}

Early specific markers of hepatocytes, AFP and FOXa2, first showed expression 7 days after hepatic differentiation in vitro. The mRNA level of EZH2 decreased in the hepatocellular-induced mBMMSCs. Introduction of PLenti-eGFP-EZH2 elevated EZH2 mRNA in induced cells, and decreased the mRNA levels of AFP and FOXa2 (Figure 3). The overexpression of EZH2 using lentivirus was very high, with expression increased 9.7-fold compared with the control (Figure 3A), and an almost 8-fold decrease in AFP and FOXa2 expressions were observed at the same time (Figure 3B and C).
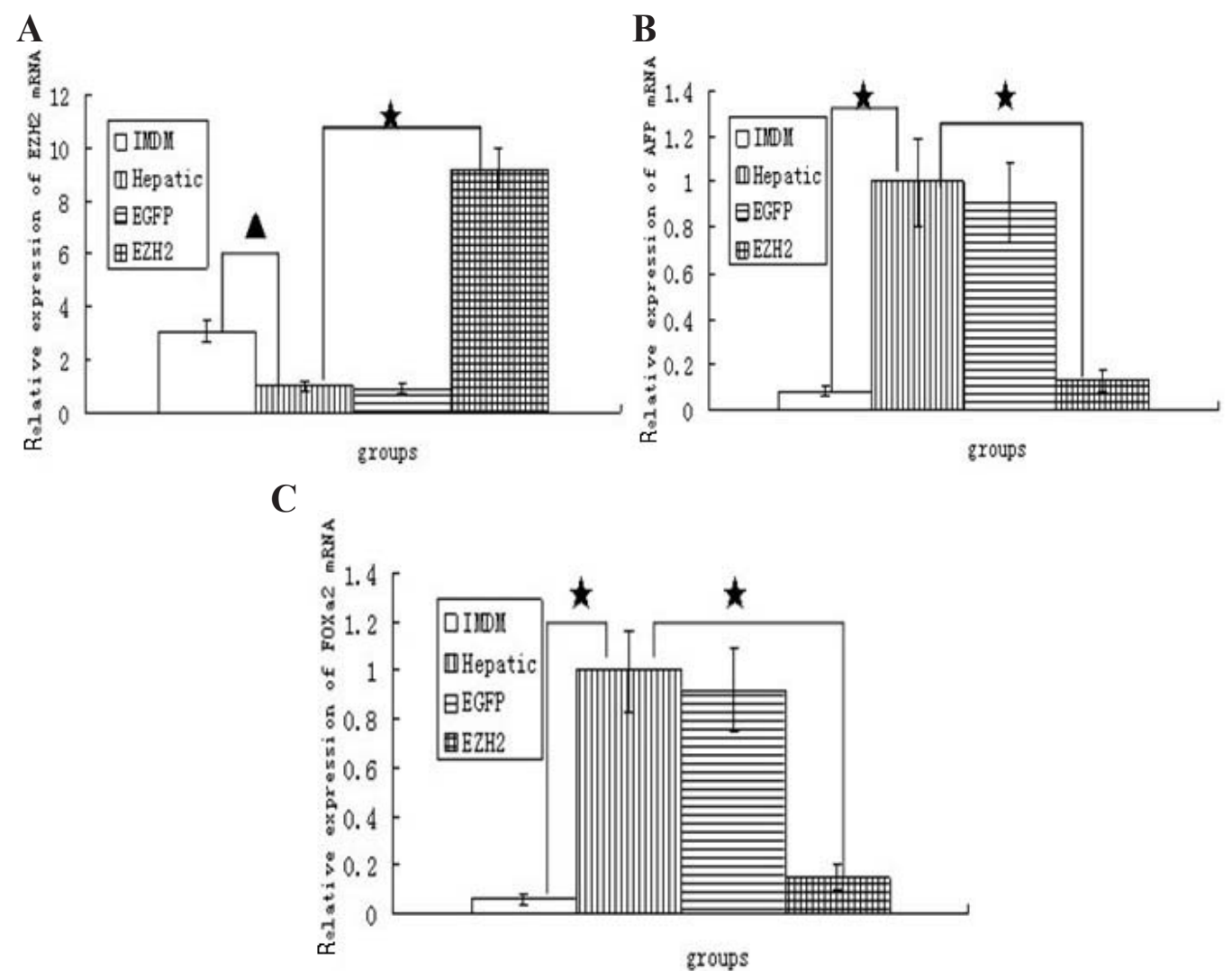

Figure 3. mRNA expression levels of genes. A. EZH2 was over-expressed by the infection of PLenti-eGFP-EZH2; the level of EZH2 mRNA in the mBMMSCs was also significantly increased compared to the hepatocellular induction group; B. and C. the expression of AFP and FOXa2 mRNA in the mBMMSCs introduced with the PLenti-eGFP-EZH2 significantly decreased compared to those with hepatocellular induction. ${ }^{\mathbf{A}} \mathrm{P}<0.05$. ${ }^{*} \mathrm{P}<0.01$.

\section{Western blot}

Introduction of PLenti-eGFP-EZH2 increased EZH2 protein expression and decreased AFP and FOXa2 protein expressions in hepatocellular-induced mBMMSCs. The EZH2 protein expression level was higher in uninduced mBMMSCs than in induced cells. The protein levels of early specific markers of hepatocytes, AFP and FOXa2, in the mBMMSCs containing 
PLenti-eGFP-EZH2 were significantly reduced compared to those of hepatocellular-induced cells (Figure 4).

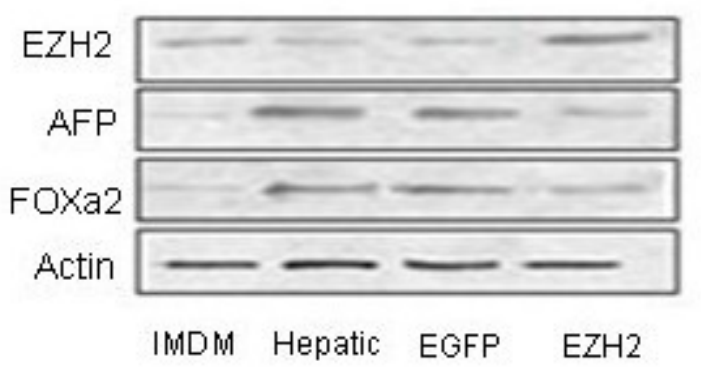

Figure 4. Protein expressions of EZH2, AFP, and FOXa2. EZH2 protein level was significantly increased by the infection of PLenti-eGFP-EZH2; it was also significantly increased in the mBMMSCs compared to the hepatocellular induction group; the protein level of early specific markers of hepatocytes, AFP and FOXa2, in the mBMMSCs that were introduced with the PLenti-eGFP-EZH2 significantly decreased compared to those of hepatocellular-induced cells.

\section{Effects of EZH2 on the level of $\mathrm{H} 3 \mathrm{~K} 27 \mathrm{me} 3$ of the $A F P$ and $F O X a 2$ promoter regions}

Hepatic priming decreased the $\mathrm{H} 3 \mathrm{~K} 27 \mathrm{me} 3$ level of the AFP and FOXa2 promoters. Introduction of EZH2 restored the level of $\mathrm{H} 3 \mathrm{~K} 27 \mathrm{me} 3$ in primed mBMMSCs (Figure 5).

A

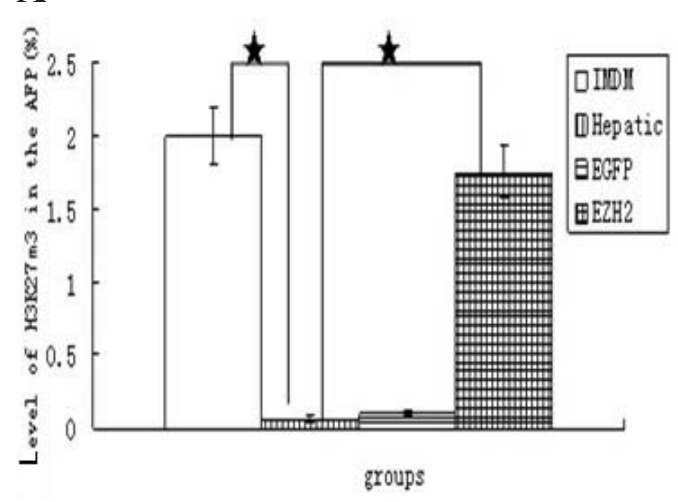

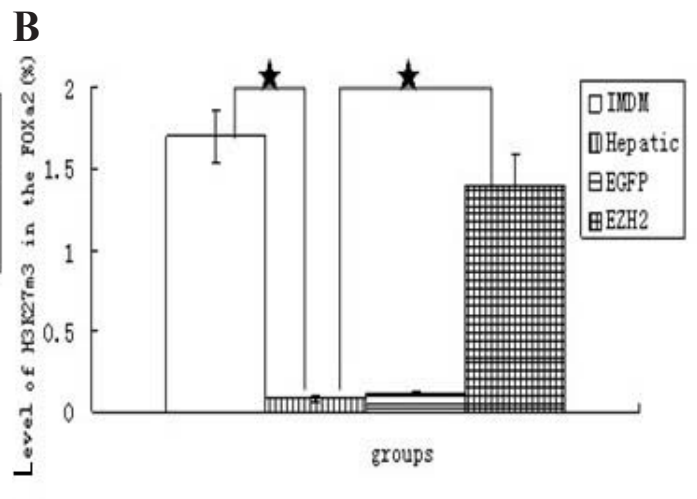

Figure 5. Effects of EZH2 on the level of H3K27me3 of AFP and FOXa2. A. Compared with the hepatocellularinduced cells, the level of $\mathrm{H} 3 \mathrm{~K} 27 \mathrm{me} 3$ of AFP was obviously increased in the uninduced mBMMSCs and in the cells with introduction of PLenti-eGFP-EZH2. B. The level of H3K27me3 of FOXa2 in each group showed a similar phenomenon.

\section{DISCUSSION}

It is believed that mBMMSCs can differentiate into many cell types of the body. Recent studies have demonstrated that BMMSCs can be induced to generate not only the progenies of 
mesodermal lineages, such as adipocytes (Prockop, 1997), chondrocytes (Li et al., 2011), and myogenic cells (Liu et al., 2010), but also cells from different germ layers, such as neuronal cells and hepatocytes (Jiang et al., 2002; Stock et al., 2010). We previously found that mBMMSCs have potential to differentiate into hepatocytes with high efficiency (Chen et al., 2006).

Epigenetics is the study of heritable properties in gene expression or cellular phenotype caused by mechanisms other than changes in the underlying DNA sequence (Ekström, 2009). The multi-lineage differentiation ability of stem cells is regulated by the expression of various genes. Indeed, increasing evidence suggests that the expression levels of related genes in undifferentiated cells is regulated by epigenetic processes of DNA and chromatin in both non-coding and coding regions (Collas, 2009). EZH2, a core component of PRC2, represses the expression of related genes by functioning as a methyltransferase for H3K27me3 (Hansen et al., 2008; Christophersen and Helin, 2010; Ezhkova et al., 2011). Furthermore, in our previous study, in vivo results indicated that the pre-treatment of the histone deacetylase inhibitor VPA significantly increased the homing efficiency of BMSSCs to the site of liver injury, and, in addition, supported hepatic differentiation in vitro (Chen et al., 2009).

In the present study, we successfully constructed PLenti-eGFP-EZH2, a lentiviral vector carrying enhanced green fluorescent protein (eGFP) and expressing the EZH2 gene. In comparison to other vectors, lentiviral vectors have higher transfection efficiencies to stem cells (Dropulić, 2011). EZH2 could be over-expressed in mBMMSCs by adding PLenti-eGFPEZH2 vector to the medium. The expression of EZH2 was higher in uninduced mBMMSCs than in hepatocellular-induced cells, which suggests that EZH2 plays an essential role in the maintenance of the self-renewal capacity of stem cells. The level of trimethylation of H3K27 also plays an important role in the differentiation of epidermal and neural stem cells (Jepsen et al., 2007; Sen et al., 2008). We discovered that the introduction of EZH2 increased the level of $\mathrm{H} 3 \mathrm{~K} 27 \mathrm{me} 3$ in the promoters of $A F P$ and $F O X a 2$ in primed mBMMSCs, which consequently lowered the expression of AFP and FOXa2. We concluded that mouse histone methyltransferase EZH2 may suppress the hepatocellular differentiation of mBMMSCs by promoting $\mathrm{H} 3 \mathrm{~K} 27 \mathrm{me} 3$ in the $A F P$ and $F O X a 2$ gene promoter regions.

Nevertheless, the epigenetic regulation mechanism in the differentiation of stem cells is very complicated, and histone methylation plays only a small part in the process. In this study, we have only revealed the role of EZH2 expression in the hepatocellular differentiation of mBMMSCs. The differentiation rate of hepatocyte-like cells from MSCs is still not high enough, and the mechanisms are also not well known. In the future, we could generate EZH2$\mathrm{KO}$ mice to increase the efficiency of hepatocyte differentiation and terminal maturation. The development of a high-efficiency hepatocyte differentiation mBMMSC model would be very useful for both basic stem cell research and clinical studies.

\section{ACKNOWLEDGMENTS}

Research supported by the Zhejiang Natural Science Foundation (\#Y2090337) and the Key Disciplines at the Zhejiang Provincial and ShaoXing city level (\#GJSX-010-003).

\section{REFERENCES}

Boyer LA, Plath K, Zeitlinger J, Brambrink T, et al. (2006). Polycomb complexes repress developmental regulators in murine embryonic stem cells. Nature 441: 349-353. 
Cao R, Wang L, Wang H, Xia L, et al. (2002). Role of histone H3 lysine 27 methylation in Polycomb-group silencing. Science 298: 1039-1043.

Chen Y, Dong XJ, Zhang GR, Shao JZ, et al. (2006). Transdifferentiation of mouse BM cells into hepatocyte-like cells. Cytotherapy 8: 381-389.

Chen Y, Dong XJ, Zhang GR, Shao JZ, et al. (2007). In vitro differentiation of mouse bone marrow stromal stem cells into hepatocytes induced by conditioned culture medium of hepatocytes. J. Cell. Biochem. 102: 52-63.

Chen Y, Pan RL, Zhang XL, Shao JZ, et al. (2009). Induction of hepatic differentiation of mouse bone marrow stromal stem cells by the histone deacetylase inhibitor VPA. J. Cell. Mol. Med. 13: 2582-2592.

Christophersen NS and Helin K (2010). Epigenetic control of embryonic stem cell fate. J. Exp. Med. 207: 2287-2295.

Collas P (2009). Epigenetic states in stem cells. Biochim. Biophys. Acta 1790: 900-905.

Dropulić B (2011). Lentiviral vectors: their molecular design, safety, and use in laboratory and preclinical research. Hum. Gene Ther. 22: 649-657.

Ekström TJ (2009). Epigenetic control of gene expression. Biochim. Biophys. Acta 1790: 845-846.

Ezhkova E, Lien WH, Stokes N, Pasolli HA, et al. (2011). EZH1 and EZH2 cogovern histone H3K27 trimethylation and are essential for hair follicle homeostasis and wound repair. Genes Dev. 25: 485-498.

Hansen KH, Bracken AP, Pasini D, Dietrich N, et al. (2008). A model for transmission of the H3K27me3 epigenetic mark. Nat. Cell Biol. 10: 1291-1300.

Jepsen K, Solum D, Zhou T, McEvilly RJ, et al. (2007). SMRT-mediated repression of an H3K27 demethylase in progression from neural stem cell to neuron. Nature 450: 415-419.

Jiang Y, Jahagirdar BN, Reinhardt RL, Schwartz RE, et al. (2002). Pluripotency of mesenchymal stem cells derived from adult marrow. Nature 418: 41-49.

Kamminga LM, Bystrykh LV, de Boer A, Houwer S, et al. (2006). The Polycomb group gene Ezh2 prevents hematopoietic stem cell exhaustion. Blood 107: 2170-2179.

Li JW, Guo XL, He CL, Tuo YH, et al. (2011). In vitro chondrogenesis of the goat bone marrow mesenchymal stem cells directed by chondrocytes in monolayer and 3-dimetional indirect co-culture system. Chin. Med. J. 124: 3080-3086.

Liu TY, Dai H, Lin J, Li M, et al. (2010). Expressions of myogenic markers in skeletal muscle differentiation of human bone marrow mesenchymal stem cells. Zhongguo Yi Хие Ке Хие Yиап Хие Вао 32: 516-520.

Pan RL, Chen Y, Xiang LX, Shao JZ, et al. (2008). Fetal liver-conditioned medium induces hepatic specification from mouse bone marrow mesenchymal stromal cells: a novel strategy for hepatic transdifferentiation. Cytotherapy 10: 668-675.

Prockop DJ (1997). Marrow stromal cells as stem cells for nonhematopoietic tissues. Science 276: 71-74.

Sen GL, Webster DE, Barragan DI, Chang HY, et al. (2008). Control of differentiation in a self-renewing mammalian tissue by the histone demethylase JMJD3. Genes Dev. 22: 1865-1870.

Stock P, Bruckner S, Ebensing S, Hempel M, et al. (2010). The generation of hepatocytes from mesenchymal stem cells and engraftment into murine liver. Nat. Protoc. 5: 617-627.

Zhang M, Wang F, Kou Z, Zhang Y, et al. (2009). Defective chromatin structure in somatic cell cloned mouse embryos. J. Biol. Chem. 284: 24981-24987. 